The Astrophysical Journal, 612:1140-1146, 2004 September 10

(C) 2004. The American Astronomical Society. All rights reserved. Printed in U.S.A.

\title{
A DETAILED STUDY OF OPACITY IN THE UPPER ATMOSPHERE OF PROXIMA CENTAURI
}

\author{
D. J. Christian, M. Mathioudakis, and D. S. Bloomfield \\ Department of Pure and Applied Physics, Queen's University Belfast, Belfast, BT7 1NN, Northern Ireland, UK \\ J. DupuIs \\ Department of Physics and Astronomy, Johns Hopkins University, 3400 North Charles Street, Baltimore, MD 21218 \\ AND \\ F. P. KEENAN \\ Department of Pure and Applied Physics, Queen's University Belfast, Belfast, BT7 1NN, Northern Ireland, UK \\ Received 2004 January 30; accepted 2004 May 26
}

\begin{abstract}
We present far-UV and UV spectroscopic observations of Proxima Centauri obtained as part of our continued investigation into the optically thin approximation assumed for the transition regions of late-type stars. Significant opacity is found in the $\mathrm{C}$ III lines during both the quiescent and flaring states of Proxima Cen, with up to $70 \%$ of the expected flux being lost in the latter. Our findings cast some doubt on the suitability of the C III $\lambda 977$ line for estimating the electron density in stellar atmospheres. However, the opacity has no significant effect on the observed line widths. We calculate optical depths for homogeneous and inhomogeneous geometries and estimate an electron density of $6 \times 10^{10} \mathrm{~cm}^{-3}$ for the transition region using the $\mathrm{O}$ iv line ratios at $1400 \AA$. The combination of electron density and optical depth indicates path lengths as low as $\approx 10 \mathrm{~km}$, which are in excellent agreement with estimates of the small-scale structure seen in the solar transition region.
\end{abstract}

Subject headings: atomic data — stars: activity — stars: atmospheres — stars: individual (GJ 551) — stars: late-type - ultraviolet: stars

\section{INTRODUCTION}

It is usually assumed that emission lines from stellar and solar transition regions and coronae are optically thin. Deviations from optically thin conditions and opacity effects have been known for many years in the solar corona, but only recently have space-borne instruments with higher sensitivity and resolution allowed this assumption to be tested in other stars. Spectroscopic observations carried out with the Extreme Ultraviolet Explorer (EUVE; $\lambda / \Delta \lambda \sim 300$ ) showed a low lineto-continuum ratio, which was taken as evidence of resonant scattering in the corona of cool stars (Schrijver et al. 1994). Follow-up studies with instruments of higher resolution and sensitivity on board XMM-Newton and Chandra have found no significant opacity effects for a large sample of late-type stars (Ness et al. 2003). The excess continuum flux seen by EUVE was most likely due to a large number of lines not accounted for in the plasma codes (Jordan 1996). Spectrographs available on the Hubble Space Telescope (HST) and the Far Ultraviolet Spectroscopic Explorer (FUSE) have provided the necessary resolution and sensitivity to investigate opacity effects in stellar transition regions (Mathioudakis et al. 1999; Bloomfield et al. 2002).

In this paper we present a detailed study of the well-known flare star Proxima Centauri (dM5.5e; GJ 551). At a distance of $1.3 \mathrm{pc}$, Proxima Cen is the closest star to the Sun. Its low rotation rate (period $\approx 83$ days, Benedict et al. 1998) is combined with a deep convection zone (fully convective) to generate the intense flare activity seen on the star. Its proximity, combined with the increased flare activity, have made Proxima Cen a prime target for the study of flares in X-rays. Such observations by XMM-Newton have revealed continuously varying "quiescent" X-ray flux and flares spanning 3 orders of magnitude in energy (Güdel et al. 2004). The dimensions of these flares are comparable to the stellar radius, while electron densities reached values as high as $4 \times 10^{11} \mathrm{~cm}^{-3}(T \approx 2 \mathrm{MK})$.

In $\S 2$ we present the FUSE and HST observations and analysis. Light curves and temporal parameters for the flare events observed by FUSE are presented for two different emission lines. In $\S 3$ we present the results from the FUSE and HST Space Telescope Imaging Spectrometer (STIS) analysis. These include the spectra observed with FUSE and STIS, and the ratios of line fluxes for emission lines of interest. In $\S 4$ we present the optical depths derived from the ratio of emission-line fluxes. These, combined with the values of the electron density, allow us to calculate the extent of the scattering regions. Last, in $\S 5$ we present the conclusions of our findings.

\section{OBSERVATIONS AND ANALYSIS}

We observed Proxima Cen with FUSE on 2003 April 5 and 6 (program D122). The observations were made with the low-resolution aperture (LWRS) in time-tagged mode. FUSE consists of four co-aligned prime-focus telescopes, with two telescopes having $\mathrm{SiC}$ coatings and optimized for the 905$1105 \AA$ region and the others using $\mathrm{LiF}$ coatings to cover the 987-1187 ^ region. Holographically ruled gratings disperse light onto two separate microchannel plate detectors (MCPs), each with two independent segments. The spectral resolution for the LWRS aperture is $\approx 12,000-15,000$. Details on the FUSE instrument and in-orbit performance can be found in Moos et al. (2000) and Sahnow et al. (2000a, 2000b).

FUSE data were reduced with the currently available version (ver. 2.4) of the CalFUSE pipeline. Spectra were extracted from the LWRS, and these were background subtracted, flatfielded, and wavelength and flux calibrated as described in the CalFUSE reference guide (van Dixon et al. 2001). The 


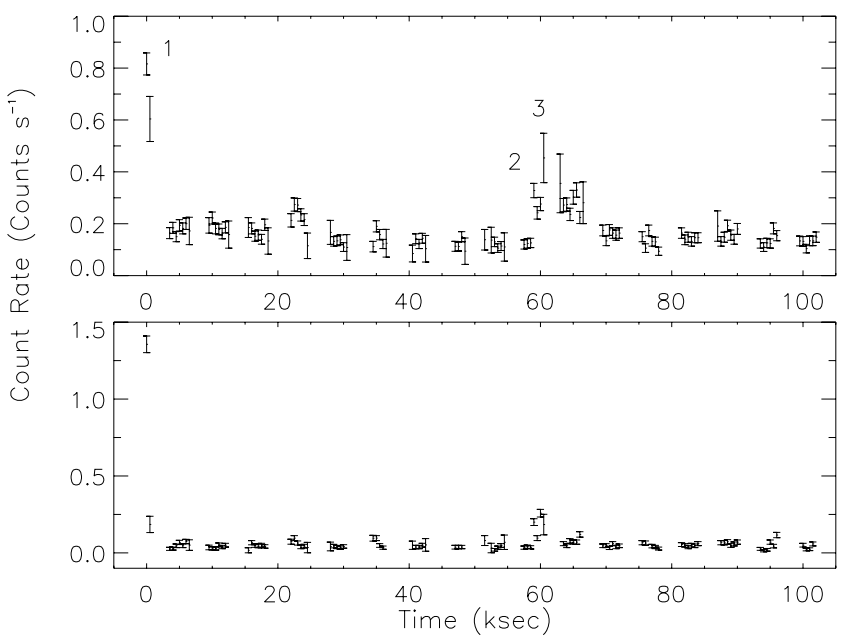

FIG. 1.-Proxima Cen light curves for the entire FUSE observation. The top panel shows the $\mathrm{O}$ VI $\lambda 1032$ light curve and the bottom panel shows the $\mathrm{C}$ III $\lambda 1176$ multiplet light curve. The start time for the first bin is MJD 52734.4748, and the bin size is $500 \mathrm{~s}$ for both light curves. Flares 1, 2, and 3 are noted in the top panel.

observations covered 19 satellite orbits. Timing analysis revealed a flare in the first orbit and two smaller flares twothirds of the way through the observations. Spectra were extracted for each flare and labeled 1 to 3 . Light curves for the entire observation extracted in the lines of $\mathrm{O}$ VI $\lambda 1032$ and the total C III $\lambda 1176$ multiplet are shown in Figure 1. The flares are discussed in more detail below.

We have combined spectra from different detector channels to increase the signal-to-noise ratio. This was accomplished using the FUSE_REGISTER ${ }^{1}$ tool provided by the FUSE project. Combined spectra for the wavelength regions of interest are segments $1 \mathrm{BSiC}$ and $2 \mathrm{ASiC}$ for $\mathrm{C}$ III 2977 , segments $1 \mathrm{ALiF}$ and $1 \mathrm{ASiC}$ for $\mathrm{O}$ vi $\lambda 1032$ and $\mathrm{O}$ vi $\lambda 1038$, and segments $2 \mathrm{ALiF}$ and $1 \mathrm{BLiF}$ for the $\mathrm{C}$ III $\lambda 1176$ multiplet. All spectra were binned to a 4 pixel resolution, which corresponds to $\sim 8 \mathrm{~km} \mathrm{~s}^{-1}$, approximately half of a formal resolution element.

We have also obtained archival STIS data for Proxima Cen. This star was observed on 2000 May 8 as part of program 8280 (PI Linsky) in medium-resolution echelle mode $(R \sim 40,000)$ with the E140M grating covering 1150-1710 $\AA, 0.2 \times 0.2$ aperture, and MAMA detector. During seven successful orbits, noted as data set $05 \mathrm{eo} 0$, data were accumulated for a total of $41 \mathrm{ks}$. One of these orbits revealed a small flare. Flux-calibrated spectra were extracted from each of the six quiescent orbits in the emission lines of $\mathrm{N} v \lambda 1240$, Si IV $\lambda 1400$, and $\mathrm{C}$ IV $\lambda 1550$ and combined using the HST_CALIB.STIS IRAF routines.

We derived line fluxes by fitting Gaussians to the observed line profiles. Although a small red component may be seen in the profiles of some of the lines, we found that single Gaussians were sufficient to fit the FUSE O vI and C III transitions. However, two Gaussian components were used to fit the $\mathrm{N} v$, Si IV, and $\mathrm{C}$ IV lines observed with STIS. In addition, the six-component $\mathrm{C}$ III $\lambda 1176$ multiplet was fitted with five separate curves, with the $\lambda \lambda 1175.59$ and 1175.71 lines being treated as a single feature. The central wavelength of each Gaussian was required to have the same spacing as the laboratory measurements and linked to the $\lambda 1174.9$ line, but the

\footnotetext{
${ }^{1}$ FUSE_REGISTER and other FUSE IDL tools are available at http:// fuse.pha.jhu.edu/analysis/fuse_idl_tools.html.
}

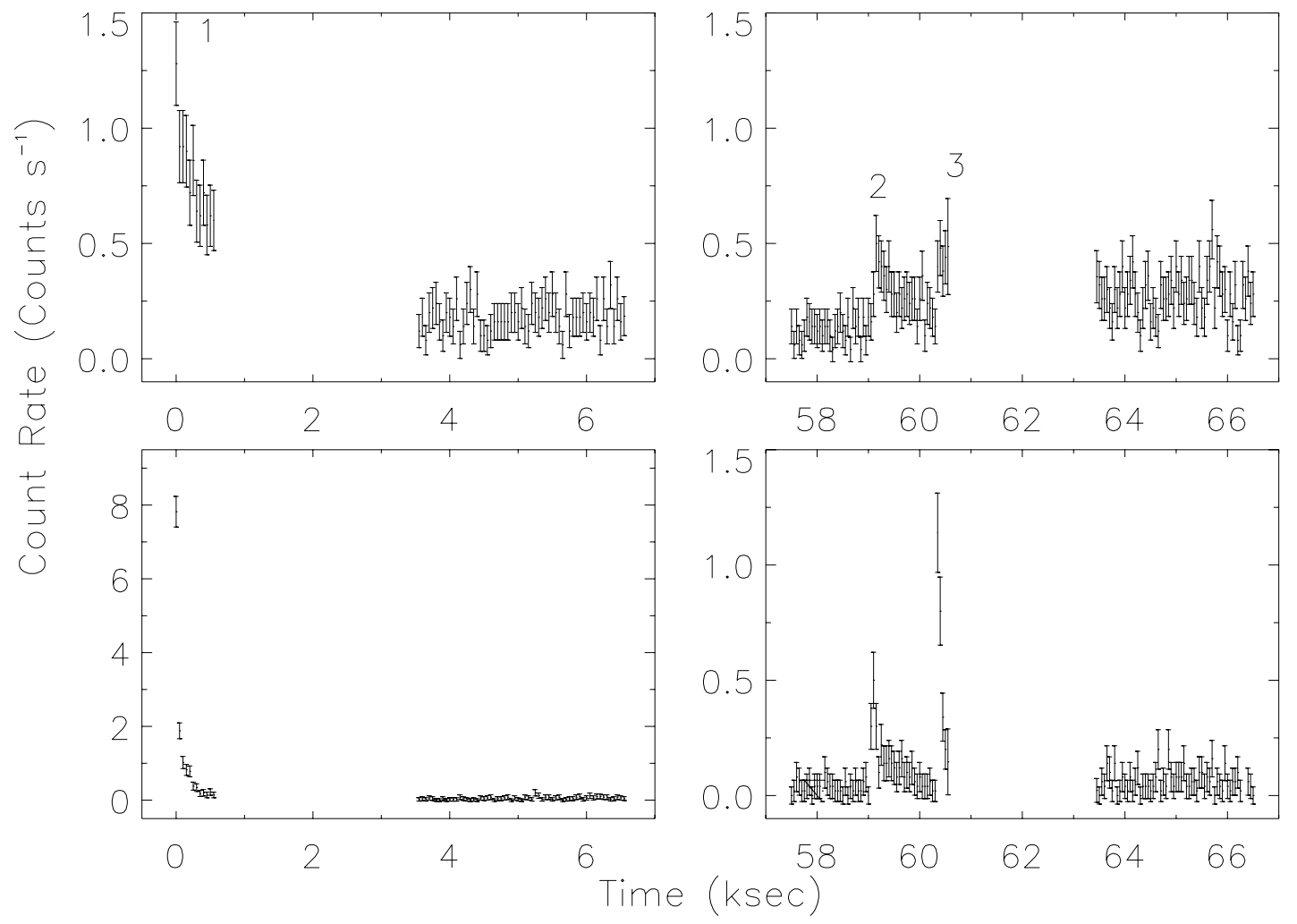

FIg. 2.-Expanded light curves of the flares from Proxima Cen, showing flare 1 on the left and flares 2 and 3 on the right. As in Fig. 1, the top panel shows the $\mathrm{O}$ VI $\lambda 1032$ light curve and the bottom panel shows the $\mathrm{C}_{\text {III }} \lambda 1176$ multiplet light curve. The bin size is $50 \mathrm{~s}$ for all light curves. 
TABLE 1

FUSE Light-Curve Results

\begin{tabular}{|c|c|c|c|c|c|c|c|c|c|c|c|}
\hline \multirow[b]{2}{*}{ Flare } & \multirow[b]{2}{*}{$\mathrm{MJD}^{\mathrm{a}}$} & \multicolumn{5}{|c|}{ O VI $\lambda 1031.9$} & \multicolumn{5}{|c|}{$\mathrm{C}$ III $\lambda 1176$} \\
\hline & & $\begin{array}{c}\text { Peak }^{\mathrm{b}} \\
\left(\text { counts s}^{-1}\right)\end{array}$ & Max/Mean ${ }^{c}$ & $\begin{array}{l}t_{\text {rise }} \\
(\mathrm{s})\end{array}$ & $\begin{array}{l}t_{\text {decay }} \\
(\mathrm{s})\end{array}$ & $\begin{array}{c}e \text {-fold }^{\mathrm{d}} \\
(\mathrm{s})\end{array}$ & 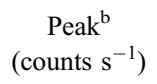 & Max/Mean ${ }^{c}$ & $\begin{array}{l}t_{\text {rise }} \\
(\mathrm{s})\end{array}$ & $\begin{array}{l}t_{\text {decay }} \\
(\mathrm{s})\end{array}$ & $\begin{array}{c}e-\text { fold }^{\mathrm{d}} \\
(\mathrm{s})\end{array}$ \\
\hline Flare $1 \ldots \ldots$ & 52734.4748 & $1.28 \pm 0.18$ & 8.4 & $\ldots$ & $>600$ & 80 & $7.8 \pm 0.4$ & 40 & $\ldots$ & $>600$ & 80 \\
\hline Flare $2 \ldots . .$. & 52735.1594 & $0.50 \pm 0.12$ & 3.3 & 150 & 1000 & 800 & $0.5 \pm 0.12$ & 3 & 200 & 900 & 500 \\
\hline Flare $3 \ldots \ldots$ & 52735.1739 & $0.48 \pm .012$ & 3.1 & 300 & $>250$ & $>400$ & $1.14 \pm 0.17$ & 6 & $<50$ & $>200$ & 40 \\
\hline
\end{tabular}

a MJD of the flare peak.

b Peak count rate of the flare.

c Ratio of the maximum flare count rate (peak) to the average quiescent rate.

d $e$-folding time of the flare decay.

wavelength of the latter was left as a free parameter. In this way any shifts caused by the spectrometers could be taken into account. The profile widths were set to be equal for all lines, but the actual value was left as a free parameter.

Expanded light curves for the flares observed in the $\mathrm{O}$ VI $\lambda 1032$ and $\mathrm{C}$ III $\lambda 1176$ lines are shown in Figure 2. The rise for the first flare, flare 1 , was before the start of our observation, and the decay was interrupted by the first FUSE occultation. Flares 1 and 2 lasted for 600 and $1200 \mathrm{~s}$, respectively. The temporal properties of all three flares are summarized in Table 1. Flare 1 showed a remarkable rise in the $\mathrm{C}$ III $\lambda 1176$ line flux, which increased by a factor of $\approx 40$ over the quiescent level.

The luminosities of the flares range from $9 \times 10^{25}$ to $2.3 \times$ $10^{26} \mathrm{ergs} \mathrm{s}^{-1}$ for $\mathrm{C}$ III $\lambda 1176$, and $2 \times 10^{25}$ to $10^{26} \mathrm{ergs} \mathrm{s}^{-1}$ for $\mathrm{O}$ vi $\lambda 1032$.

\section{RESULTS}

\subsection{Opacity from Line Ratios}

The optical depth at line center is given by

$$
\tau_{0}=1.16 \times 10^{-14} \lambda f_{i j} \sqrt{\frac{M}{T}} \frac{n_{i}}{n_{\mathrm{el}}} \frac{n_{\mathrm{el}}}{n_{\mathrm{H}}} \frac{n_{\mathrm{H}}}{n_{e}} n_{e} l,
$$

where $\lambda$ is the wavelength in angstroms, $f_{i j}$ the oscillator strength of the transition, $M$ the mass of the absorbing atom in atomic mass units, $T$ the temperature in kelvins, $l$ the path length in centimeters and $n_{i}, n_{\mathrm{el}}, n_{\mathrm{H}}$, and $n_{e}$ are the number densities in cubic centimeters of ions in the lower level $i$, the element, hydrogen, and free electrons, respectively (Mitchell \& Zemansky 1961). In Table 2 we summarize the atomic parameters for the transitions of the ions used in this work. Line ratios from these transition are used to derive line optical depths. The $\mathrm{C}_{\text {III }} \lambda \lambda 1175.71$ and 1174.94 emission lines arise from a common upper level, and their expected line flux ratio scales as the ratio of their Einstein $A$-values. However, the $\mathrm{O}$ vI, N v, Si Iv, and $\mathrm{C}$ Iv ion emission line pairs have a common lower level, and their expected line flux ratio scales as the ratio of their oscillator strengths. For a detailed description of the method used the reader is referred to Bloomfield et al. (2002).

In Figure 3, C III $\lambda 1176$ spectra for flare 1 and the quiescent state of Proxima Cen are shown. The ratio of the $\mathrm{C}_{\text {III }} \lambda 1175.7$ to the $\mathrm{C}$ III $\lambda 1174.9$ line is expected to be 3 in optically thin conditions. These line ratios are predicted to show no $n_{e}$ or $T_{e}$ dependence over the $10^{6}-10^{14} \mathrm{~cm}^{-3}$ and $10^{4}-10^{7} \mathrm{~K}$ ranges (CHIANTI database; Dere et al. 1997). However, C III ratios deviate from the optically thin approximation in all three flares observed, as well as in the quiescent state. Flares 1 and 3 show the largest deviations, with ratios of $1.68 \pm 0.14$ and $1.57 \pm$ 0.25 , respectively. We have calculated relative line strengths for the transitions of the $\mathrm{C}$ III multiplet using a model ion consisting of the 10 energetically lowest fine-structure levels (see Bloomfield et al. 2002 for details). By scaling from the $\lambda 1174.93$ line, which we assume to be optically thin, we can estimate the total flux expected for the $\lambda 1176$ multiplet in optically thin conditions. We find that $80 \%$ of the expected $\mathrm{C}$ III $\lambda 1176$ flux is observed during quiescence, while $70 \%$ and

TABLE 2

Ion Parameters and the Line Transitions Used in the Present Study

\begin{tabular}{|c|c|c|c|c|c|c|c|c|c|}
\hline Ion $(\AA)$ & $\log T$ & $M_{\mathrm{el}}$ & $n_{\text {ion }} / n_{\mathrm{el}}$ & $\begin{array}{c}n_{\mathrm{el}} / n_{\mathrm{H}} \\
\left(\times 10^{-4}\right)\end{array}$ & $\begin{array}{c}\lambda \\
(\AA)\end{array}$ & Transition & $f_{i j}^{\mathrm{a}}$ & $\begin{array}{c}A_{j i}^{\mathrm{a}} \\
\left(10^{8} \mathrm{~s}^{-1}\right)\end{array}$ & $\tau_{\text {rel }}$ \\
\hline \multirow[t]{2}{*}{ 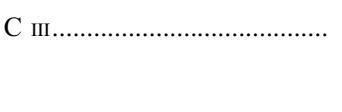 } & 4.8 & 12.01 & 0.865 & 3.98 & $977.02^{\mathrm{b}}$ & $2 s^{2}{ }^{1} S_{0}-2 s 2 p{ }^{1} P_{1}$ & 0.81 & $\ldots$ & 29.31 \\
\hline & 4.8 & 12.01 & 0.865 & 3.98 & $1174.94^{\mathrm{c}}$ & $2 s 2 p{ }^{3} P_{1}-2 p^{2}{ }^{3} P_{2}$ & 0.11 & 3.1 & 1.69 \\
\hline \multirow[t]{2}{*}{$\mathrm{O}$ VI } & 5.5 & 16.00 & 0.239 & 8.51 & $1031.91^{\mathrm{b}}$ & $2 s^{2} S_{1 / 2}-2 p^{2} P_{3 / 2}$ & 0.131 & $\ldots$ & $\ldots$ \\
\hline & 5.5 & 16.00 & 0.239 & 8.51 & $1037.61^{\mathrm{c}}$ & $2 s^{2} S_{1 / 2}-2 p^{2} P_{1 / 2}$ & 0.065 & $\ldots$ & $\ldots$ \\
\hline \multirow[t]{2}{*}{ Si IV } & 4.8 & 28.10 & 0.350 & 1.3 & $1393.76^{\mathrm{b}}$ & $3 s^{2} S_{1 / 2}-3 p{ }^{2} P_{3 / 2}$ & 0.52 & $\ldots$ & $\ldots$ \\
\hline & 4.8 & 28.10 & 0.350 & 1.3 & $1402.77^{\mathrm{c}}$ & $3 s^{2} S_{1 / 2}-3 p{ }^{2} P_{1 / 2}$ & 0.26 & $\ldots$ & $\ldots$ \\
\hline \multirow[t]{2}{*}{ C IV } & 5.0 & 12.01 & 0.350 & 3.9 & $1548.20^{\mathrm{b}}$ & $2 s^{2} S_{1 / 2}-2 p{ }^{2} P_{3 / 2}$ & 0.20 & $\ldots$ & $\ldots$ \\
\hline & 5.0 & 12.01 & 0.350 & 3.9 & $1550.77^{\mathrm{c}}$ & $2 s^{2} S_{1 / 2}-2 p^{2} P_{1 / 2}$ & 0.10 & $\ldots$ & $\ldots$ \\
\hline
\end{tabular}

${ }^{\text {a }} f_{i j}$ and $A_{j i}$ values from Wiese et al. (1966).

$\mathrm{b}$ Optically thick line.

${ }^{c}$ Optically thin line. 


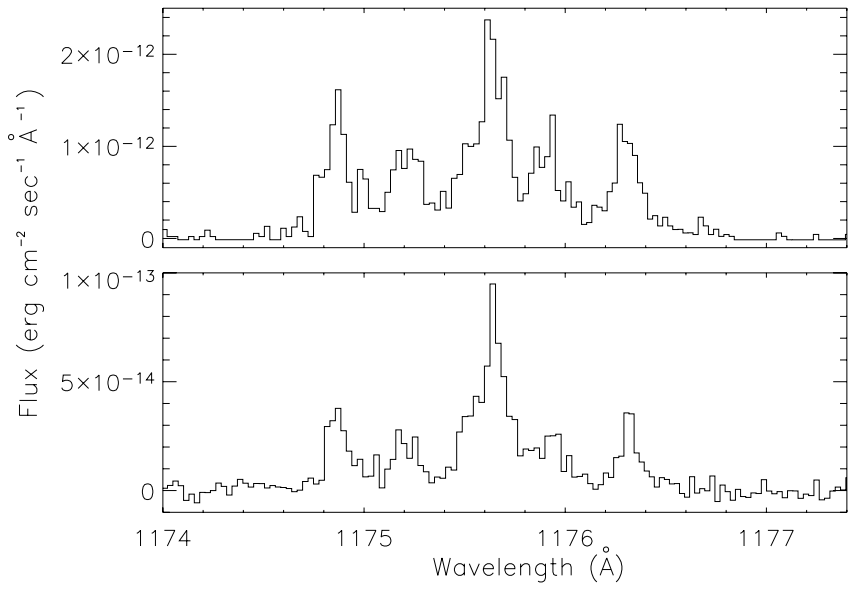

FIG. 3.-FUSE co-added C III $\lambda 1176$ multiplet spectra. The top panel shows the spectrum during flare 1 , and the bottom panel shows the quiescent spectrum.

$60 \%$ of the expected flux is observed for flares 1 and 3 , respectively. If we assume a density of $10^{11} \mathrm{~cm}^{-3}$, we find that $\approx 70 \%$ of the expected C III $\lambda 977$ flux is observed during quiescence and only $30 \%$ during flare 1 .

In Figure 4 we show sample spectra for the $O$ VI $\lambda \lambda 1032$ and 1038 resonance lines. For the optically thin case, the ratio of oscillator strengths for these transitions is 2 . There is no evidence for significant opacity in $\mathrm{O}$ VI with the observed ratios of $1.85 \pm 0.11$ for the quiescent spectrum and $2.25 \pm 0.15$ for flare 1. The deviation for the $\mathrm{O}$ vi ratio for flare $3(1.3 \pm 0.2)$ is not deemed to be statistically significant because of the low signal-to-noise ratio in this spectrum. Line fluxes and derived ratios are shown for the quiescent and flaring spectra in Table 3.

The observed $\mathrm{Si}$ IV and $\mathrm{N} v$ ratios $(\approx 2.0 \pm 0.1)$ are also consistent with the theoretical ratios, while a small deviation from optically thin conditions is detected in C IV (1.75 \pm 0.04$)$. The STIS fluxes and line ratios are listed in Table 4.

If a certain geometry is assumed, the observed ratios can be used to estimate escape probabilities and optical depths for the lines under study. We consider both a homogeneous distribution of emitters and absorbers and an inhomogeneous distribution (Kastner \& Kastner 1990; Bloomfield et al. 2002). In the latter case, the emitters and absorbers are spatially distinct. Table 3 presents the optical depths derived from the two distributions, with those derived from the homogeneous approximation being a factor of 2-3 times larger. We derive constraints on the extent of the scattering region for both distributions in $\S 4$.

\subsection{Opacity and Line Widths}

Opacity can also affect the widths of spectra lines, as photons absorbed at line center can be re-emitted in the wings. Kastner \& Bhatia (1997) have studied this problem extensively and derived equations that relate the half-width at halfmaximum of a Doppler profile with the optical depth. The observed FWHM of the C III $\lambda 1176$ lines is $\Delta \lambda_{\text {obs }}=0.21 \pm$ $0.01 \AA$. This is essentially the convolution of thermal, nonthermal, opacity, and instrumental broadening. Taking into account that the FUSE instrumental broadening has a Gaussian profile $\Delta \lambda_{\text {inst }}=0.067 \AA$ (G. Williger 2004, private communication), the deconvolved stellar width is $\Delta \lambda_{\text {stell }} \approx 0.20 \AA$. Proxima Cen is a very slow rotator, and hence rotational broadening is insignificant. Redfield et al. (2002) have studied the nonthermal velocities in the transition region of stars with convective envelopes. Their results show a trend where highgravity stars have lower nonthermal velocities compared to

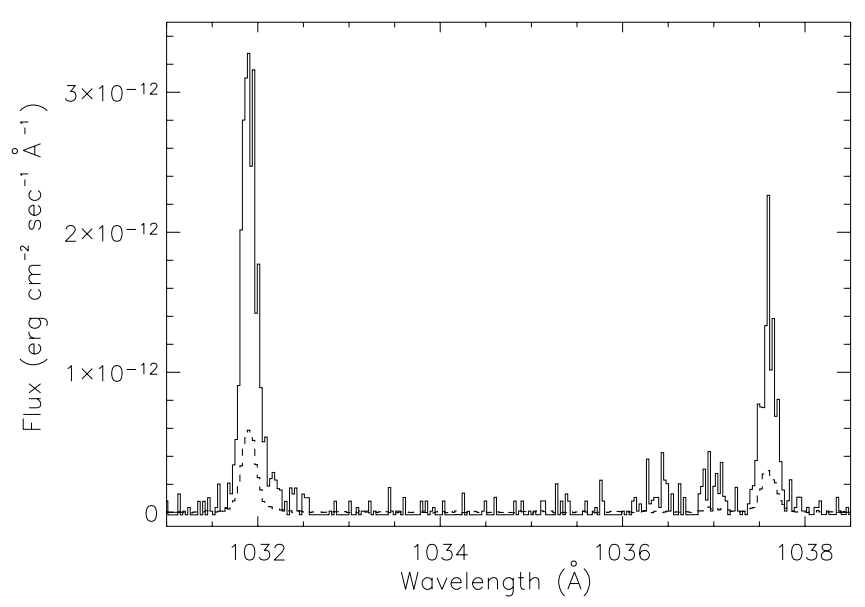

FIG. 4.-FUSE co-added spectra of the O VI resonance lines. The solid plot is the observation taken during flare 1 (containing both flare and quiescent components), while the dashed plot was taken during quiescence.

stars of lower gravity. Using the lines of the C III multiplet, they derive a nonthermal velocity $\xi$ of $26 \pm 9 \mathrm{~km} \mathrm{~s}^{-1}$ for AU Mic. If we assume the same nonthermal velocity for Proxima Cen, and also take into account thermal broadening, we find a Doppler FWHM of $\Delta \lambda \approx 0.18 \AA$. The intrinsic line width can therefore be explained by these two processes. We note that the $\mathrm{C}$ III $\lambda 977$ line, with a much higher optical depth, has essentially the same FWHM as the $\lambda 1176$ transition. Although there is a significant increase in the optical depth between the quiescent state and flare 1 , there is no increase in the any of the observed line widths. We therefore conclude that opacity has no significant effect on the observed line widths. We show sample C III $\lambda 977$ spectra for quiescence and flare 1 in Figure 5.

\section{DISCUSSION}

\subsection{Line Ratio Constraints on Emission Size}

The derived optical depths now allow us to place constraints on the extent of the scattering region with the only unknown quantity being the electron density (eq. [1]). CHIANTI (Dere et al. 1997) allows us to calculate theoretical line ratios for the ions of interest. For $\mathrm{C}$ III, the ratio of the total emission of the $\lambda 1176$ multiplet to that of the $\mathrm{C}$ III $\lambda 977$ line has a density dependence in the $10^{8}$ to $5 \times 10^{10} \mathrm{~cm}^{-3}$ range. We find a $\mathrm{C}$ III $\lambda 1176 / \lambda 977$ ratio of $0.54 \pm 0.03$ in the quiescent spectrum. This value is at the high density limit $n_{e}>10^{11} \mathrm{~cm}^{-3}$ for a temperature of $\log T=4.74$. If a higher temperature is adopted $(\log T=4.92)$ this would give a density of $\approx 3 \times 10^{10} \mathrm{~cm}^{-3}$. Similar densities have been found in the quiescent atmospheres of Altair and Procyon (Redfield et al. 2002). In the flare spectra of Proxima Cen this ratio is greater than 1 , which again implies a density $n_{e}>10^{11} \mathrm{~cm}^{-3}$. However, as mentioned earlier the $\mathrm{C}$ III $\lambda 977$ line is optically thick, and any diagnostics that use this line provide only a lower limit to the density.

The O IV lines near $1400 \AA$ fall in the same STIS order as Si IV and are a powerful density diagnostic at higher temperatures $(\log T=5.1)$. Both the $O$ IV $\lambda 1399.78 / \lambda 1404.79$ and $\lambda 1407.38 / \lambda 1404.79$ ratios are density sensitive in the range $\approx 10^{10}-10^{12} \mathrm{~cm}^{-3}$. From the calculations of Keenan et al. (2002), we find that the observed $\lambda 1399.78 / \lambda 1404.79$ ratio is $1.46 \pm 0.29$, which implies an electron density of $\approx 7 \times 10^{10} \mathrm{~cm}^{-3}$. The ratio of $\lambda 1407.38 / \lambda 1404.79$ is $0.94 \pm$ 0.23 , implying a density of $4 \times 10^{10} \mathrm{~cm}^{-3}$. Averaging these, 
TABLE 3

Line Fluxes and Ratios of the C iil Multiplet and O vi Resonance Lines

\begin{tabular}{|c|c|c|c|c|c|c|c|c|c|c|c|c|}
\hline \multirow{4}{*}{$\begin{array}{l}\text { IoN } \\
(\AA)\end{array}$} & \multicolumn{6}{|c|}{$\mathrm{C}$ III } & \multicolumn{6}{|c|}{$\mathrm{O}$ VI } \\
\hline & \multicolumn{2}{|c|}{ Flux } & \multirow[b]{3}{*}{ Ratio } & \multirow[b]{3}{*}{$P_{\text {thick }} / P_{\text {thin }}$} & \multirow{2}{*}{\multicolumn{2}{|c|}{$\tau_{1175.7}$}} & \multicolumn{2}{|c|}{ Flux } & \multirow[b]{3}{*}{ Ratio } & \multirow[b]{3}{*}{$P_{\text {thick }} / P_{\text {thin }}$} & \multirow{2}{*}{\multicolumn{2}{|c|}{$\tau_{1031.9}$}} \\
\hline & 1175.7 & 1174.9 & & & & & 10319 & 10376 & & & & \\
\hline & $\left(10^{-14} \mathrm{ergs} \mathrm{cm}^{-2} \mathrm{~s}^{-1}\right)$ & $\left(10^{-14} \mathrm{ergs} \mathrm{cm}^{-2} \mathrm{~s}^{-1}\right)$ & & & Hom. & Inh. & $\left(10^{-14} \mathrm{ergs} \mathrm{cm}^{-2} \mathrm{~s}^{-1}\right)$ & $\left(10^{-14} \mathrm{ergs} \mathrm{cm}^{-2} \mathrm{~s}^{-1}\right)$ & & & Hom. & Inh. \\
\hline Quiet............ & $1.6(0.1)$ & $0.6(0.1)$ & $2.56(0.28)$ & 0.84 & 0.81 & 0.38 & $12.1(0.3)$ & $6.6(0.3)$ & $1.85(0.11)$ & 0.91 & 0.57 & 0.27 \\
\hline Flare $1 \ldots \ldots$ & $42.9(0.2)$ & $25.6(0.2)$ & $1.68(0.14)$ & 0.55 & 4.28 & 1.43 & $55.0(1.5)$ & $24.4(1.5)$ & $2.25(0.15)$ & 1.11 & & \\
\hline Flare $2 \ldots \ldots$ & $4.7(0.4)$ & $2.1(0.4)$ & $2.25(0.43)$ & 0.74 & 1.54 & 0.52 & $9.7(0.5)$ & $4.1(0.6)$ & $2.40(0.35)$ & 1.19 & $\ldots$ & .. \\
\hline Flare $3 \ldots \ldots$ & 16.0 & $10.2(1.4)$ & $1.57(0.25)$ & 0.52 & 5.16 & 1.59 & 16.9 & 12.8 & $1.32(0.17)$ & 0.65 & 4.3 & 1.35 \\
\hline
\end{tabular}

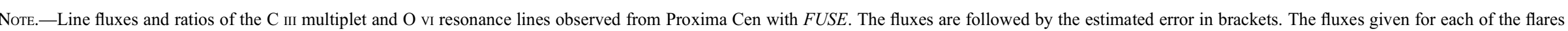
are the total observed fluxes minus the quiescent components. 
TABLE 4

Line Fluxes and Ratios of the C iv, Si iv, and N v Doublets Observed with STiS

\begin{tabular}{|c|c|c|c|c|c|}
\hline \multirow[b]{2}{*}{ EMISSION LiNE } & \multirow{2}{*}{$\begin{array}{c}\text { FluX } \\
\left(10^{-14} \mathrm{ergs} \mathrm{cm}^{-2} \mathrm{~s}^{-1}\right)\end{array}$} & \multirow[b]{2}{*}{ RATIO } & \multirow[b]{2}{*}{$P_{\text {thick }} / P_{\text {thin }}$} & \multicolumn{2}{|c|}{$\tau_{\text {ion }}$} \\
\hline & & & & Hom. & Inh. \\
\hline $\mathrm{N}$ v $\lambda 1238.7 \ldots \ldots \ldots \ldots \ldots$ & $2.51(0.06)$ & & & .. & .. \\
\hline $\mathrm{N}$ v $\lambda 1242.8 \ldots \ldots \ldots \ldots . . .$. & $1.27(0.03)$ & $1.98(0.10)$ & 0.99 & $\ldots$ & . \\
\hline Si IV $\lambda 1393.8 \ldots \ldots \ldots \ldots . . . . .$. & $1.31(0.07)$ & & & $\cdots$ & . \\
\hline Si IV $\lambda 1402.8 \ldots \ldots \ldots \ldots \ldots$ & $0.65(0.03)$ & $2.01(0.10)$ & 1.00 & $\ldots$ & .. \\
\hline C Iv $\lambda 1548.2 \ldots \ldots \ldots \ldots . . .$. & $7.5(0.1)$ & & & 0.79 & 0.36 \\
\hline $\mathrm{C}$ iv $\lambda 1550.8 \ldots \ldots \ldots \ldots$ & $4.28(0.08)$ & $1.75(0.04)$ & 0.88 & $\ldots$ & $\ldots$ \\
\hline
\end{tabular}

we have therefore adopted a value of $6 \times 10^{10} \mathrm{~cm}^{-3}$ for our calculations.

We show a comparison of the observed spectra and theoretical line profiles in Figure 6. Line profiles were generated with the CHIANTI spectral synthesis package for the above derived density, and a solar flare differential emission measure (DEM) for the flare spectra and the solar quiet DEM for quiescent spectra. The opacity effects for the $C_{\text {III }} \lambda 1175.71$ (top panel) and C IV 21548.2 (bottom panel) lines are clearly visible.

We use the electron density derived from $\mathrm{O}$ IV and the optical depths (eq. [1]) to derive values or upper limits to the effective size of the scattering regions. In quiescence, we derive $\operatorname{Hom}_{\mathrm{C} \text { III }} \approx 13 \mathrm{~km}$ and $\mathrm{Inh}_{\mathrm{C} \text { II }} \approx 7 \mathrm{~km}$ path lengths for homogeneous and inhomogeneous geometries, respectively. A comparison of the STIS line fluxes with earlier IUE observations (Haisch et al. 1983) confirms that they were observed at quiescence in the STIS observations. $\mathrm{C}$ IV is the only line ratio that deviates from the optically thin approximation. The extent of the scattering region derived from the $\mathrm{C}$ IV ion are $\mathrm{Hom}_{\mathrm{C} \text { IV }} \approx 30 \mathrm{~km}$ and $\mathrm{Inh}_{\mathrm{C} \text { IV }} \approx 15 \mathrm{~km}$. These $\mathrm{C}$ III path lengths are comparable to those determined for the quiescent state of AU Mic (Bloomfield et al. 2002).

During the FUSE flares, the optical depths have increased by up to a factor of 6 ; hence, the physical extent is larger by this amount. The path lengths are summarized in Table 5.

We derive a volume emission measure $\left(\mathrm{EM}=n_{e}^{2} V\right)$ from the observed line fluxes and emissivities of Doyle \& Keenan (1992). If we combine the EM with the electron density, we find $V \approx 10^{27} \mathrm{~cm}^{3}$ and $V \approx 2 \times 10^{26} \mathrm{~cm}^{3}$ for $\mathrm{C}$ III and $\mathrm{O}$ VI, respectively. This implies filling factors of approximately $10^{-4}$

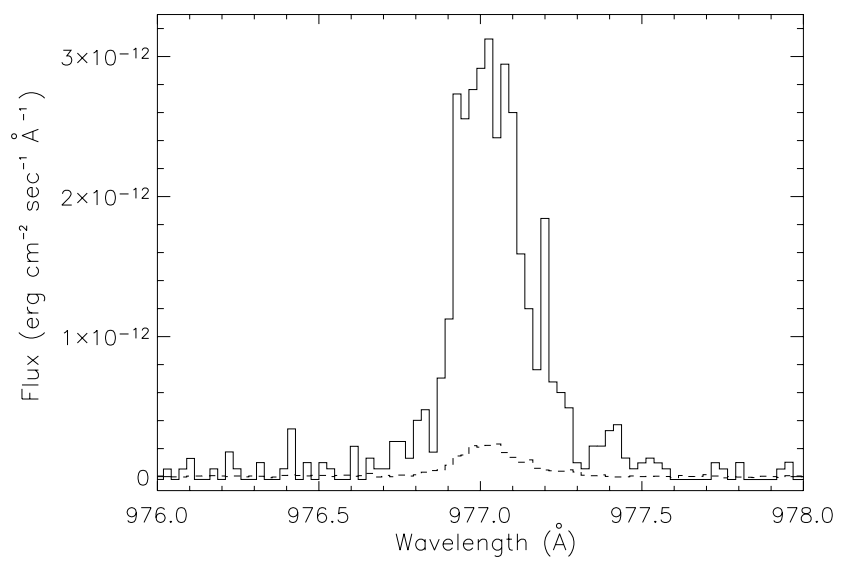

FIG. 5.-Same as Fig. 4, but for the C III 2977.02 resonance line. to $10^{-5}$. If we assume that the emission originates in a large single loop or a series of $N$ loops with loop radius $R$ and crosssectional radius $r$, the volume is $V=0.04 \pi^{2} R^{3} N$ for $r \approx$ $0.2 R$. For a single loop this implies loop radii of $\approx 10,000 \mathrm{~km}$. If instead we assume 100 identical loops contributing to the emission, the radius $R$ is $\approx 2000 \mathrm{~km}$. The derived radii for flares 2 and 3 are $\approx 30 \%$ smaller. We emphasize that although the assumption of isothermal loops may be a good approximation for some parts of the corona, the solar transition region emission arises from inhomogeneous structures in the chromosphere-corona interface and this assumption may not necessarily be applicable, but it does provide a simplified loop estimate.

\section{CONCLUSIONS}

We study the effects of opacity in the transition region of the active late-type star Proxima Cen. Our analysis reveals significant deviations of the line fluxes from the optically thin approximation, particularly in the case of the $\mathrm{C}_{\text {III }} \lambda \lambda 977$ and 1176 lines. These deviations become more pronounced during flares. Lines affected by opacity appear weaker compared to both the continuum and other transitions. This must be taken into account when these lines are used as density diagnostics or are employed in the construction of EM distributions. An examination of line widths reveals no significant opacity

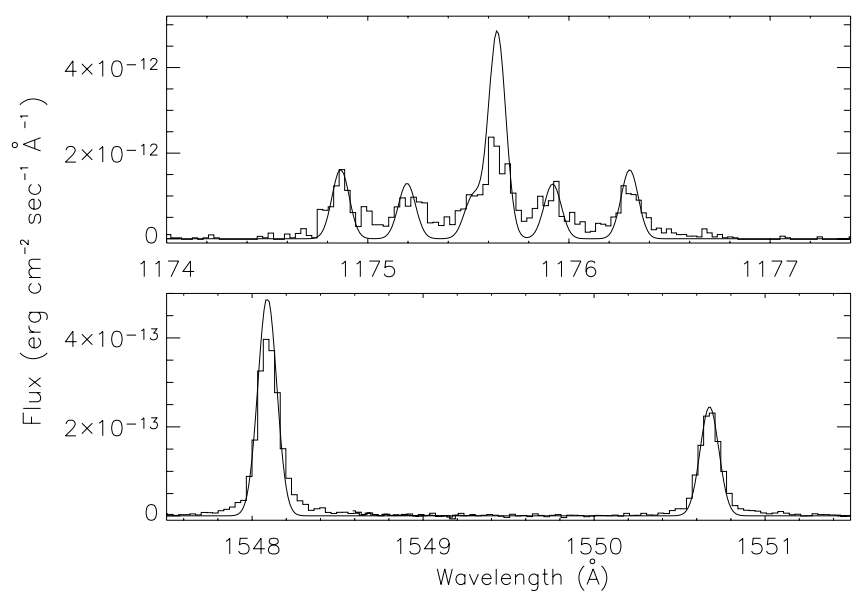

FIG. 6.-Theoretical line profiles (solid line) overplotted on the observed spectra (solid histogram). The top panel shows the FUSE C III $\lambda 1176$ spectrum during flare 1, and the bottom panel shows the STIS C Iv spectrum during quiescence. The line profiles were generated with CHIANTI using our derived density $\left(6 \times 10^{10} \mathrm{~cm}^{-3}\right)$ and solar flare and quiescent DEMs for the top and bottom panels, respectively (see text). 
TABLE 5

Table of Derived Path Lengths for Observed Ions

\begin{tabular}{cccr}
\hline \hline \multirow{2}{*}{ Ion } & & \multicolumn{2}{c}{ PATH LENGTH } \\
\cline { 3 - 4 } & & $\begin{array}{c}\text { Hom. } \\
(\mathrm{km})\end{array}$ & $\begin{array}{r}\text { Inh. } \\
(\mathrm{km})\end{array}$ \\
\hline $\mathrm{O}$ VI ................ & Flare 3 & 380 & 120 \\
$\mathrm{C}$ III................. & Quiet & 13 & 6 \\
& Flare 1 & 70 & 22 \\
& Flare 2 & 24 & 8 \\
& Flare 3 & 80 & 25 \\
& $\ldots$ & 30 & 15 \\
\hline
\end{tabular}

Note.-The $\mathrm{O}$ vi quiescent, $\mathrm{N} v$, and $\mathrm{Si}$ Iv ratios are optically thin within the derived uncertainties.

broadening; the FWHM is dominated by thermal and nonthermal broadening.

Opacity can also be used as a diagnostic tool, as the optical depth is proportional to the line-of-sight path length. Hence, optical depths combined with independent measurements of the electron density allow direct estimates of the path lengths in the transition region. In the example studied here, we find path lengths in the range of $10-100 \mathrm{~km}$. These path lengths are very similar to those reported in a recent study of the solar transition region (Doschek \& Feldman 2004). Solar and stellar transition regions must therefore have similar spatial characteristics.

This research was partially supported by NASA FUSE Guest Investigator grant NNG04GC75G. We thank the FUSE Mission Operations Team for their skilled execution of this observation, and we thank Gerard Williger for information on the FUSE line-spread functions. F. P. K. is grateful to A. W. E. Aldermaston for the award of a William Penney Fellowship. Data sets presented here were obtained from the Multimission Archive at the Space Telescope Science Institute (MAST). STScI is operated by the Association of Universities for Research in Astronomy, Inc., under NASA contract NAS526555. Support for MAST for non-HST data is provided by the NASA Office of Space Science via grant NAG5-7584 and by other grants and contracts.
Benedict, G., et al. 1998, AJ, 116, 429

Bloomfield, D. S., Mathioudakis, M., Christian, D. J., Keenan, F. P., \& Linsky, J. 2002, A\&A, 390, 219

Dere, K. P., Landi, E., Mason, H. E., Monsignori-Fossi, B. C., \& Young, P. R. 1997, A\&AS, 125, 149

Doschek, G. A., \& Feldman, U. 2004, ApJ, 600, 1061

Doyle, J. G., \& Keenan, F. P. 1992, A\&A, 264, 173

Güdel, M., Audard, M., Reale, F., Skinner, S. L., \& Linsky, J. L. 2004, A\&A, 416, 713

Haisch, B. M., Linsky, J. L., Bornmann, P. L., Stencel, R. E., Antiochos, S. K., Golub, L., \& Vaiana, G. S. 1983, ApJ, 267, 280

Jordan, C. 1996, in IAU Colloq. 152, Astrophysics in the Extreme Ultraviolet, ed. S. Bowyer \& R. F. Malina (Boston: Kluwer), 81

Kastner, S. O., \& Bhatia, A. K. 1997, J. Quant. Spectrosc. Radiat. Transfer, 58,217

Kastner, S. O., \& Kastner, R. E. 1990, J. Quant. Spectrosc. Radiat. Transfer, 44, 275

Keenan, F. P., et al. 2002, MNRAS, 337, 901

\section{REFERENCES}

Mathioudakis, M., McKenny, J., Keenan, F. P., Williams, D. R., \& Phillips, K. J. H. 1999, A\&A, 351, L23

Mitchell, A. C. G., \& Zemansky, M. W. 1961, Resonance Radiation and Excited Atoms (Cambridge: Cambridge Univ. Press)

Moos, H. W., et al. 2000, ApJ, 538, L1

Ness, J.-U, Schmitt, J. H. M. M., Audard, M., Güdel, M., \& Mewe, R. 2003, A\&A, 407, 347

Redfield, S., Linsky, J. L., Ake, T. B, Ayres, T. R., Dupree, A. K., Robinson,

R. D., Wood, B. E, \& Young, P. R 2002, ApJ, 581, 626

Sahnow, D. J., et al. 2000a, ApJ, 538, L7 2000b, Proc. SPIE, 4013, 334

Schrijver, C. J., van den Oord, G. H. J., \& Mewe, R. 1994, A\&A, 289, L23

Van Dixon, W., Kruk, J., \& Murphy, E. 2001, CalFUSE Reference Guide (Baltimore: Johns Hopkins Univ.), http://fuse.pha.jhu.edu/analysis/calfuse .html

Wiese, W. L., Smith, M. W., \& Glennon, B. M. 1966, Atomic Transition Probabilities (Washington: GPO) 